\title{
Need for follow up in coeliac disease
}

\author{
M T Bardella, N Molteni, L Prampolini, A M Giunta, A R Baldassarri, D Morganti, \\ P A Bianchi
}

Department of
Gastroenterology,
Istituto di Scienze
Mediche, Milan, Italy
M T Bardella
N Molteni
A R Baldassarri
D Morganti
P A Bianchi
Department of
Paediatrics, University
of Milan, Milan, Italy
L Prampolini
A M Giunta
Correspondence to:
Dr M T Bardella, Cattedra
di Gastroenterologia, Istituto
di Scienze Mediche,
Padiglione Granelli, Via
Francesco Sforza, 35, 20122
Milan, Italy.
Accepted 1 December 1993

\begin{abstract}
The use of follow up studies was evaluated in 128 patients with coeliac disease during their first visit to a department for adults. The original diagnosis had been made in childhood in all patients. Fifty eight $(45 \%)$ of the subjects were following a gluten free diet, $23(18 \%)$ were following a gluten free diet but with occasional gluten consumption, and $47(37 \%)$ had adopted an unrestricted, gluten containing diet for a mean of 11.2 years. There was no correlation in individual subjects between the presence of symptoms, biochemical and immunological abnormalities, severity of histological findings, and the amount of dietary gluten, despite the greater frequency of symptoms in the group following an unrestricted diet than in the other two groups. Short stature and epilepsy with cerebral calcifications only occurred in patients following an unrestricted diet. As only diagnosis based on two or three biopsy samples and regular follow up correlated positively with dietary compliance, it is suggested that a histologically confirmed diagnosis of coeliac disease and regular lifelong follow up are essential in the management of these patients.

(Arch Dis Child 1994; 70: 211-213)
\end{abstract}

Coeliac disease is an enteropathy characterised by mucosal damage of the small intestine by dietary gluten. Until recently gluten intolerance was considered to be permanent, and lifelong abstention is thought to be necessary to prevent nutritional deficiencies and to avoid the late risks associated with coeliac disease. ${ }^{1}$ Follow up studies on dietary compliance, however, generally show it to be poor in the long term. ${ }^{2} 3$

The aim of our study was to evaluate the use of follow up studies in relation to compliance with a gluten free diet and the occurrence of symptomatic, biochemical, or morphological relapses in a group of teenagers and young adults during their first visit to a department for adults after a diagnosis of coeliac disease in childhood.

\section{Patients and methods}

We studied 128 patients ( 55 men, 73 women) with a median age of 18 years (range 11-30) referred to us by the University of Milan department of paediatrics or by the patients' general practitioners in the period 1972-92.
Diagnosis was based on the European Society of Pediatric Gastroenterology and Nutrition (ESPGAN) criteria in 75 patients, biopsy samples before and after gluten withdrawal in three, biopsy samples before gluten withdrawal and clinical response to a gluten free diet in 18, and clinical grounds and response to a gluten free diet in 32 . Three of the patients were older than 3 years when coeliac disease was diagnosed and 12 were older than 10 years. All 32 clinical diagnoses were made when the patients were less than 2 years old. The diagnosis was confirmed in all patients by two biopsy samples with and without gluten in their diet taken in our department, if not previously in childhood.

A detailed dietary inquiry was carried out and compared with the separate views of the patients and their parents on the strictness of their diet. If the gluten free diet had been abandoned, the length of time that they had been on normal diet and the factors affecting the decision to change were recorded. Patients were allocated to one of the following three groups based on the interview and their diet in the previous year and without a knowledge of laboratory findings: group A, subjects following a strict gluten free diet; group $B$, subjects following a gluten free diet with occasional gluten consumption (from one to two gluten containing foods each month to one each week); and group C, subjects following a normal (unrestricted) diet.

The patients' height and weight were recorded, as were the presence of symptoms or signs of malabsorption or associated diseases, or both, a family history of coeliac disease, and the frequency of previous examinations. Height was considered to be abnormally low when less than the fifth centile for chronological age and sex, if growth was yet not complete, and when less than the fifth centile for chronological age and sex corrected for mid-parental height, if growth was complete. Weight was considered abnormal when less than $10 \%$ of the ideal body weight, evaluated on weight for height charts and adjusted for sex and age according to the Italian National Institute of Nutrition tables (revision 1986-7). The following blood tests were performed: haemoglobin, blood cell counts, total serum proteins and albumin, serum calcium, phosphate, and alkaline phosphatase, serum iron, and vitamin B-12 and folic acid. Liver and renal function tests were performed. Specific IgA antibodies to gliadin were measured with the Pharmacia Gluten IgA EIA kit (upper normal level 25 arbitrary units, AU). 
Table 1 Occurrence of clinical, biochemical, and histological abnormalities in 128 teenagers and young adults with coeliac disease receiving different diets (group $A$, gluten free diet; group $B$, occasional gluten consumption; group $C$, unrestricted diet)

\begin{tabular}{|c|c|c|c|}
\hline & $\begin{array}{l}\text { Group } A \\
(n=58)\end{array}$ & $\begin{array}{l}\text { Group } B \\
(n=23)\end{array}$ & $\begin{array}{l}\text { Group } C \\
(n=47)\end{array}$ \\
\hline \multicolumn{4}{|l|}{ No of patients with symptoms } \\
\hline Tiredness & & 4 & 12 \\
\hline Diarrhoea & & & 9 \\
\hline Abdominal pain & & & 3 \\
\hline Dyspepsia & & & 3 \\
\hline Mouth apthae & & & 1 \\
\hline Short stature & & & 5 \\
\hline Underweight & & & 5 \\
\hline Allergic rhinitis or asthma & & & 3 \\
\hline Tetany & & 1 & 1 \\
\hline Epilepsy & & & 5 \\
\hline Acute relapse & & & 9 \\
\hline Total No $(\%)$ of patients & 0 & $5^{\star}(21)$ & $25(53)$ \\
\hline \multicolumn{4}{|l|}{$\begin{array}{l}\text { No of patients with biochemical abnormalities } \\
\text { Haemoglobin }(<2.17 \mathrm{mmol} / \mathrm{l} \text { (males) }\end{array}$} \\
\hline$-<1.86 \mathrm{mmol} / \mathrm{h}$ (females) & 3 & 10 & 15 \\
\hline Low serum iron $(<13 \mu \mathrm{mol} / 1)$ & 3 & 9 & 32 \\
\hline Low plasma calcium $(<2.25 \mathrm{mmol} / \mathrm{l})$ & & 1 & 8 \\
\hline IgA antigliadin antibody $(>25 \mathrm{AU})$ & & 6 & 27 \\
\hline \multicolumn{4}{|l|}{ High serum transaminases (more than twice } \\
\hline $\begin{array}{l}\text { upper normal value) } \\
\text { Total No (\%) of patients }\end{array}$ & 4 (7) & & 3 \\
\hline \multicolumn{4}{|l|}{ No of patients with histological abnormalities } \\
\hline Grade I-II abnormalities & & 1 & 4 \\
\hline Grade III abnormalities & & 3 & $i$ \\
\hline Grade IV abnormalities & & & 42 \\
\hline Total No (\%) of patients & $7(12)$ & $6(26)$ & $47(100)$ \\
\hline
\end{tabular}

${ }^{\star} \chi^{2}=20 \cdot 61, \mathrm{p}<0.001 v$ group $\mathrm{C} ;{ }^{\star \star} \chi^{2}=96 \cdot 22, \mathrm{p}<0 \cdot 001 v$ group $\mathrm{C}$.

Table 2 Diagnosis and follow up in 128 teenagers and young adults with coeliac disease according to diet compliance (group $A$, gluten free diet; group $B$, occasional gluten consumption; group $C$, unrestricted diet)

\begin{tabular}{lllll}
\hline & $\begin{array}{l}\text { No of } \\
\text { patients }\end{array}$ & $\begin{array}{l}\text { Group } \\
A\end{array}$ & $\begin{array}{l}\text { Group } \\
B\end{array}$ & $\begin{array}{l}\text { Group } \\
C\end{array}$ \\
\hline ESPGAN or two biopsy & & & & \\
$\quad$ samples & 78 & $58^{\star}$ & 16 & 4 \\
One biopsy sample & 18 & 0 & 6 & 12 \\
No biopsy sample & 32 & 0 & 1 & 31 \\
Yearly follow up & 98 & $58^{\star \star}$ & 21 & 19 \\
No follow up & 30 & 0 & 2 & 28 \\
\hline
\end{tabular}

${ }^{\star} \chi^{2}=92 \cdot 16, \mathrm{p}<0.001 v$ groups $\mathrm{B}$ and $\mathrm{C}$ considered together.

$\star \star \chi^{2}=54 \cdot 74, \mathrm{p}<0.001$ v groups $B$ and $C$ considered together.

Repeat distal mucosal duodenal biopsy samples were taken during an endoscopic examination in 56 patients; specimens were stained with haematoxylin-eosin and periodic acid-Schiff and histological features were classified according to Scott and Losowsky. ${ }^{4}$

\section{Results}

Table 1 gives the clinical, biochemical, and histological abnormalities of our patients and table 2 their compliance with a gluten free diet according to grounds for diagnosis and follow up.

Group A (following a gluten free diet) consisted of $58(45 \%)$ patients, 24 men and 34 women, median age 17 years (range 11-22); diagnosis was based on ESPGAN criteria in 55 patients and on two biopsy samples in the other three. These subjects had been seen regularly each year in the department of paediatrics and could consult a psychologist and dietician as necessary. They were all well and had no clinical problems. Three women $(15,16$, and 22 years) had serum iron concentrations of between 5.37 and $8.95 \mu \mathrm{mol} / 1$. Seven subjects, including two girls with sideropenic anaemia, had a jejunal biopsy sample taken and the histology was normal in all samples. Only one patient had an associated disease, dermatitis herpetiformis, diagnosed at 5 years of age together with coeliac disease. Six patients had a family history of coeliac disease.

In group B (occasional gluten consumption) there were $23(18 \%)$ patients, 13 men and 10 women, median age 18 years (range 15-27). The diagnosis was based on ESPGAN criteria or two biopsy samples in 16 patients; six had only one biopsy sample taken and one no biopsy sample. Twenty one had been followed up regularly, like the patients in group A. They were aware of their dietary indiscretions, which mostly occurred outside their homes at social events. Of the remaining two patients one was followed up for the first few years on a gluten free diet after a clinical diagnosis of coeliac disease, but did not return to hospital until the age of 24 years when she was admitted with severe anaemia. The other patient, who had had juvenile diabetes mellitus since the age of 5 years, did not accept the diet and follow up when coeliac disease was diagnosed at the age of 13 years; she was severely ill with bone and muscular pain, cheilitis, anaemia, osteoporosis, and altered blood tests when she arrived at our department aged 18 years. IgA antibody for gliadin was positive in six patients, and did not correlate with clinical or biochemical findings or with histology. Biopsy samples from six subjects (three positive for IgA antibody to gliadin) all showed histological alterations, grade II in one patient, grade III in three, and grade IV in two. No associated disease was observed other than diabetes. Only one patient had a family history of coeliac disease.

Forty seven $(37 \%)$ patients were in group C, 18 men and 29 women, median age 19 years (range 13-30). Only four had a diagnosis based on three or two biopsy samples, 12 had one biopsy sample taken, and in 31 the diagnosis was made clinically without an intestinal biopsy sample. In 28 patients the parents, sometimes on the advice of their general practitioner, had tried other foods and finally abandoned the gluten free diet because of the wellbeing of the children. None of these patients had been followed up by the hospital where the diagnosis was made. The mean duration following an unrestricted diet at their arrival in our department was 16.2 years (range 11-25). The other 19 subjects in this group were followed up after diagnosis. They gradually suspended the gluten free diet of their own accord because of wellbeing, and completely abandoned it at a mean age of 18.9 years. At attendance in our department they had been following an unrestricted diet for a mean period of seven years (range 1-16). Four subjects in group $\mathrm{C}$ had a family history of coeliac disease. The five patients with short stature had been following a normal diet for more than 10 years. Epilepsy was present in five patients, with cerebral calcifications in four of them. One 30 year old asymptomatic woman had to return to a gluten free diet when pregnant because of arrested fetal development 


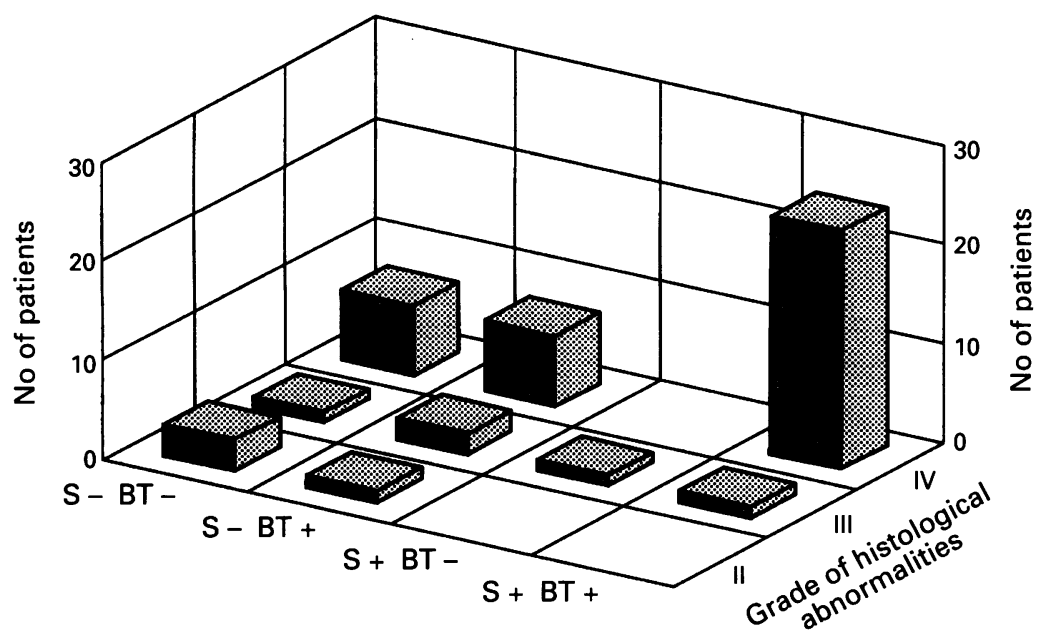

Relation between symptoms, biochemical tests, and grade of histological abnormalities in 47 patients with coeliac disease on a normal diet. $S-=$ no symptoms; $B T-=$ no biochemical abnormalities; $S+=\operatorname{symptom}(\mathrm{s})$ present; $B T+=$ biochemical abnormality (ies) present.

at five months' gestation. The relation between symptoms, biochemical tests, and grade of histological abnormalities in group $\mathrm{C}$ is shown in the figure.

In most instances there was agreement about diet compliance as assessed by doctors, parents, and patients.

\section{Discussion}

At their first visit to our department for adults $58(45 \%)$ of 128 teenagers and young adults with coeliac disease were following a strict gluten free diet. This result is similar to those reported in other studies of dietary compliance in coeliac disease. ${ }^{2356}$ In our study all the patients following a strict gluten free diet met the ESPGAN criteria or had a diagnosis based on at least two biopsy samples and were regularly followed up, whereas of the $47(37 \%)$ patients following an unrestricted diet, 31 $(66 \%)$ had a diagnosis on clinical grounds alone and $28(60 \%)$ were not followed up. Thus only diagnosis based on three or two biopsy samples and regular follow up correlated positively with diet compliance.

Our observations in patients whose diet occasionally included gluten and in those following a normal diet confirmed the known absence of correlation in individual subjects between clinical, biochemical, immunological, and histological findings and the amount of dietary gluten. ${ }^{78}$ Indeed, even though half of the group following an unrestricted diet had no symptoms, biochemical and histological findings indicated overt coeliac disease. Moreover, almost all major clinical disorders occurred in this group, including short stature and epilepsy with cerebral calcifications. Although the aetiology of calcifications is still unknown, it cannot be excluded that the lack of some micronutrients could be responsible. ${ }^{9}$ These observations emphasise the importance of the withdrawal of gluten.

It was confirmed that tests for antibody to gliadin are not useful in detecting the ingestion of small amounts of gluten. ${ }^{10}$ These tests were positive in only six $(27 \%)$ of our patients with an occasional intake of gluten and in 33 (71\%) of those following an unrestricted diet. Positive tests always correlated with the presence of histological abnormalities, however.

Like other workers, we found that the doctor's assessment of dietary compliance correlated well with histological findings, and we suggest that regular contact is essential, mainly to obtain dietary compliance but also with regard to the timing of repeat biopsy samples. ${ }^{5}$ We consider follow up to be indispensable for patients who do not comply with the diet, to detect early complications and histological relapse. In our series all the patients who had abandoned their gluten free diet had a histological relapse, which occurred after one to 16 years of following an unrestricted diet. In particular, one girl with an ESPGAN diagnosis in the first years of life had normal biopsy samples after three and six years of following an unrestricted diet, and a pathological sample three years later.

We suggest that patients who reportedly do not relapse while following an unrestricted diet should be re-evaluated to ensure that the initial diagnosis was correct and that the follow up period was sufficiently long. This would contribute to our understanding of the natural history of coeliac disease.

1 Holmes GKT, Prior P, Lane MR, Pope D, Allan RN. Malignancy in coeliac disease. Effect of gluten-free diet. Gut 1989; 30: 333-8.

2 Jackson PT, Glasglow JFT, Thom R. Parents' understanding of coeliac disease and diet. Arch Dis Child 1985; 60 $672-4$.

3 Kumar PJ, Walker-Smith J, Milla P, Harris G, Colyer J, Halliday $R$. The teenage coeliac: follow-up study of 102 patients. Arch Dis Child 1988; 63: 916-20.

4 Scott BB, Losowsky MS. Patchiness and duodenal jejunal variation of the mucosal abnormality in coeliac disease variation of the mucosal abnormality in coeliac disea

5 Congdon P, Mason MK, Smith S, Crollick A, Steel A Littlewood J. Small bowel mucosa in asymptomatic children with celiac disease. $A m$ f Dis Child 1981; 135: 118-21.

6 Mayer M, Greco L, Troncone R, Auricchio S, Marsh MN. Compliance of adolescents with coeliac disease with a gluten free diet. Gut 1991; 32: 881-5.

7 McNicholl B, Egan-Mitchell B, Fottrell PF. Variability of gluten intolerance in treated childhood coeliac disease. Gut 1979; 20: 126-32.

8 Guiraldes E, Gutierrez C. Coeliac disease and holy communion. Lancet 1988; i: 57.

9 Gobbi G, Bouquet F, Greco L, et al. Coeliac disease, epilepsy, and cerebral calcifications. Lancet 1992; ii: 439-43.

10 Troncone R, Ferguson A. Antigliadin antibodies. Review. f Pediatr Gastroenterol Nutr 1991; 12: 150-8. 